\title{
La historia laboral pensional como elemento necesario para el acceso a la pensión: el caso de Bogotá D.C.
}

The Labor Work History as Mandatory Requirement to Access to the Pension: the Case of Bogotá

Rubén Guillermo Junca-Mejía

Fondo de Prestaciones Económicas, Cesantías y Pensiones,

Colombia

DOI: https://doi.org/10.11144/Javeriana.vj137.lhlp

ORCID: http://orcid.org/0000-0002-1033-5141

Zulma Constanza Guauque-Becerra

Fondo de Prestaciones Económicas, Cesantías y Pensiones,

Colombia

ORCID: http://orcid.org/0000-0002-2864-3177

Ángela Marcela Carrillo-Gutiérrez

Fondo de Prestaciones Económicas, Cesantías y Pensiones,

Colombia

ORCID: http://orcid.org/0000-0002-5092-5760

\section{Resumen:}

Este artículo aborda el problema estructural del Sistema General de Pensiones en Colombia, en relación con la información consignada en la historia laboral pensional y cómo este problema genera contratiempos para el reconocimiento de prestaciones económicas. Tiene como objetivo principal proponer una solución a esta problemática específicamente en el caso de Bogotá D.C. Este trabajo hace parte del estudio realizado en el marco del Convenio de Cooperación 000003 de 2016 celebrado entre el Fondo de Prestaciones Económicas, Cesantías y Pensiones de Bogotá, FONCEP y la Organización Iberoamericana de Seguridad Social, OISS.

Palabras clave: Sistema General de Pensiones, historia laboral pensional, administradora de fondo de pensiones, aportes al Sistema General de Pensiones, bono pensional.

\begin{abstract}
:
This paper addresses that structural problem of the general pension system in Colombia regarding the information contained in the pension labor history, and how this problem generates setbacks at the time of the recognition of economic benefits. The main objective is to present a solution to this problem particularly the situation of Bogota. This paper is part of the study carried out within the framework of the Cooperation Agreement 000003 of 2016 celebrated between the Benefit Fund, Layoffs and Pensions (Fondo de Prestaciones Económicas, Cesantías y Pensiones de Bogotá, FONCEP) and the Ibero-American Organization of Social Security (Organización Iberoamericana de Seguridad Social, OISS).
\end{abstract}

Keywords: General pension system, Pension work history, Administrator of pension fund, Contributions to the general pension system, pension bonus.

\section{Introducción}

La seguridad social es un tema polémico a raíz de los cambios implementados por la Constitución Política de Colombia de 1991; en su preámbulo, la Asamblea Nacional Constituyente previó asegurar a los integrantes de la Nación la vida, el trabajo, la justicia y la igualdad, con el fin de garantizar un orden político, económico y social justo. En su primer artículo, en el título de los Principios fundamentales, expresó que "Colombia es un Estado Social de Derecho" ${ }^{1}$. Con ello, dieron al país un nuevo modelo de Estado con énfasis en los derechos económicos, sociales, y culturales, dirigido especialmente hacia los niños y las personas de la tercera edad, para garantizarles los servicios de la "seguridad social integral y el subsidio alimentario en caso de indigencia" ${ }^{2}$. 
Esto es desarrollado a través del preámbulo de la Ley 100 de 1993 en la cual se define al Sistema de Seguridad Social Integral ${ }^{3}$ como "El conjunto de instituciones, normas y procedimientos, de que disponen la persona y la comunidad para gozar de una calidad de vida, mediante el cumplimiento progresivo de los planes y programas que el Estado y la sociedad desarrollen para proporcionar la cobertura integral de las contingencias, especialmente las que menoscaban la salud y la capacidad económica, de los habitantes del territorio nacional, con el fin de lograr el bienestar individual y la integración de la comunidad" ${ }^{4}$.

El presente artículo se enfocará en el Sistema General de Pensiones (SGP), mediante el cual se persigue amparar a la población de las eventualidades que se presentan en el trascurso de la vida (i.e., invalidez, vejez y muerte), concretamente, en la problemática que enfrenta el Distrito Capital con respecto a la historia laboral pensional (HLP), dentro del SGP, teniendo en cuenta que el afiliado al cumplir la edad de jubilación, no logra el reconocimiento de la pensión al no contar con las semanas requeridas o con el capital ahorrado; en consecuencia, debe seguir trabajando, empezar a buscar los certificados laborales en los cuales documenten el tiempo laborado o, si la empresa no pagó los aportes correspondientes, acudir a la acción de tutela.

Debemos comprender, que el concepto de HLP que trataremos en el presente artículo es el expuesto por la Corte Constitucional en la Sentencia T-463-16, indicando que "Es un documento emitido por las administradoras de pensiones — sean públicas o privadas - que se nutre a partir de la información sobre los aportes a pensiones de cada trabajador. En ella se relaciona el tiempo laborado, el empleador -si lo tiene- y el monto cotizado. También se consignan datos específicos sobre el salario, la fecha de pago de la cotización, los días reportados e igualmente se pueden hacer anotaciones sobre cada uno de los períodos de aportes" 5 . Así mismo, la Corte Constitucional señala que en la HLP "se acompasa con la doble faceta del derecho a la información, que, por un lado, es un derecho en sí mismo y, por otro, constituye un instrumento para el ejercicio de otros derechos" 6 .

Dicho de otra manera, la HLP contiene alguna información privada sobre la vida laboral de los aportantes, así como los reportes consignados en la HLP permiten el acceso a derechos pensionales y prestacionales. Adviértase que la Corte Constitucional en la Sentencia T-079-16 manifiesta que "el esfuerzo que la pensión de vejez busca retribuir está dado, en particular por las cotizaciones obligatorias que el trabajador efectuó durante su vida laboral. Eso explica que la [HLP], es el documento que relaciona esos aportes, se convierta en una herramienta clave dentro del proceso que antecede al reconocimiento y pago de esa prestación" 7 .

Tenemos como consecuencia de lo anterior, el surgimiento para las Administradoras de Fondos de Pensiones (AFP) el deber de preservar y velar por la certeza y fidelidad de la información consignada en sus bases de datos, para que la HLP sea "precisa, clara, detallada, compresible y oportuna" ${ }^{8}$, así como la de la obligación de organizar y sistematizar la información ${ }^{9}$.

Hay que advertir, que las inconsistencias ${ }^{10}$ en la información de la HLP generan dos grandes problemáticas: 1. la generación de demoras en el tiempo del reconocimiento de las solicitudes pensionales de los afiliados, debido a que los afiliados deben por su cuenta ajustar su HLP, previo al estudio de sus derechos. 2. Congestión en el sistema judicial, porque los ciudadanos al encontrar inconsistencias en su HLP interponen demandas o acciones de tutela contra entidades públicas o privadas por los aportes no reportados a las AFP. Estas problemáticas generadas han sido estudiadas detenidamente por la Corte Constitucional y ha llevado a la declaración del Estado de Cosas Inconstitucional (ECI ${ }^{11}$ ) por el incumplimiento sistemático en el pago de pensiones que lleva a la vulneración sistemática de los derechos fundamentales por omisión.

En el caso específico del SGP colombiano, se presentan el desbordamiento en los tiempos de respuesta de las solicitudes de reconocimiento y reliquidación pensional de los afiliados, y el incumplimiento repetido de decisiones de tutela proferidas, lo que lleva a una violación sistemática de los derechos fundamentales de los ciudadanos (i.e., derecho de petición, seguridad social, acceso a la administración de justicia) por factores estructurales de las entidades reconocedoras de pensiones. 
Como quiera que no se han efectuado estudios en relación con este tema, este artículo tiene como objetivo realizar un análisis sobre la HLP y la falla estructural que se presenta en el actual sistema de información pensional. Para el abordaje de este tema, se realiza una revisión doctrinal, legal y jurisprudencial de la HLP en Colombia, para proponer al final del documento un mecanismo que permita evitar y corregir las inconsistencias que presente la HLP de los trabajadores del Distrito Capital.

\section{Evolución histórica de la historia laboral pensional}

Según la Constitución de 1991, la seguridad social en Colombia busca amparar al afiliado o su familia frente a las contingencias que impiden a la persona trabajar de manera temporal o definitiva. Así, el derecho a la pensión es elevado a derecho fundamental de amplia regulación normativa; así, la Ley 100 de 1993 señala que para poder disfrutar de una pensión, el afiliado debe acreditar unos requisitos de ley, entre ellos, el de contar con un determinado número de semanas de cotización ${ }^{12}$ o de capital ahorrado. De esta manera, concluye que se trata "de un derecho que no es gratuito, pues surge de una acumulación de cotizaciones y de tiempos de trabajo efectuados por el trabajador" 13 , por lo cual es importante estudiar la HLP, pues es el insumo que le permite al afiliado o su familia poder solicitar el reconocimiento de la pensión.

\section{La historia laboral pensional antes de Ley 100 de 1993}

La seguridad social se organiza en Colombia a finales de la década de los 40 con la creación de la Caja Nacional de Previsión Social (CAJANAL) ${ }^{14}$ y el Instituto Colombiano de Seguros Sociales ${ }^{15}$, de esta forma se dividió el sistema previsional por sectores (i.e., público ${ }^{16}$ y privado).

Como consecuencia de esta división del sistema previsional, la construcción de la HLP variaba dependiendo del sector; así para las personas que trabajaban en el sector público, la HLP se construía a partir de las certificaciones laborales expedidas por las entidades estatales. En contraste, en el sector privado (para los afiliados al Instituto de Seguros Sociales, ISS), la HLP se empezó a construir desde el 1 de enero de 1967. Así, en el período comprendido desde esa fecha hasta el 31 de diciembre de 1994, hablamos de la historia laboral tradicional o sistema tradicional ${ }^{17}$. Los aportes que efectuaban los empleadores se soportaban mediante la emisión de facturas ante el ISS, y las novedades estaban conformadas por ingresos, afiliación, cambios de salario y retiros.

\section{La autoliquidación de aportes}

Con la Constitución de 1991 se creó el Sistema General de Seguridad Social (SGSS), desarrollado mediante la Ley 100 de 1993, que creó el SGP, compuesto por el Régimen de Prima Media (RPM), administrado por el ISS, ahora Colpensiones, y por el Régimen de Ahorro Individual (RAIS), administrado por las administradoras de fondos de pensiones, AFP. Así mismo, "de conformidad con los artículos 15 y 17 de la Ley 100 de 1993, tanto la afiliación como la cotización al SGP son obligatorias para asalariados" ${ }^{18}$, posteriormente con la Ley 797 de 2003 se establece esta obligación para los contratistas ${ }^{19}$.

De la misma manera, el artículo 22 de la Ley 100 de 1993 establece que "El empleador será responsable del pago de su aporte y del aporte de los trabajadores a su servicio. Para tal efecto, descontará del salario de cada afiliado, al momento de su pago, el monto de las cotizaciones obligatorias y el de las voluntarias que expresamente haya autorizado por escrito el afiliado, y trasladará estas sumas a la entidad elegida por el trabajador, junto con las correspondientes a su aporte, dentro de los plazos que para el efecto determine 
el Gobierno" ${ }^{20}$. La responsabilidad sobre el aporte comprende, además, la obligatoriedad de reportar las novedades ${ }^{21}$.

Se establece así, la autoliquidación de aportes que consiste en que "Los empleadores deberán autoliquidar los aportes de sus trabajadores, tanto en el RAIS como en el de RPM" ${ }^{22}$. La autoliquidación de aportes se utilizó en el período comprendido entre el 01 de enero de 1995 y el 31 de diciembre de 2007. En este mecanismo, el empleador debe reportar los aportes de sus trabajadores mes a mes y las novedades. Igualmente, el trabajador independiente debía reportar sus aportes de forma mensual ${ }^{23}$.

Por otra parte, hay que tener en cuenta que antes de la Ley 100 coexistían múltiples regímenes administrados por diversas Cajas o Fondos de Previsión Social que se encargaban de otorgar pensiones, y que estas fueron reemplazadas por las nuevas AFP. Antes de la Ley 100 de 1993 era difícil acumular semanas o tiempos de cotización trabajados para diferentes empleadores o entidades, por lo cual crea la figura de los Bonos Pensionales ${ }^{24}$, los cuales "constituyen aportes destinados a contribuir a la conformación del capital necesario para financiar las pensiones de los afiliados al SGP” 25 .

Una de las características del Bono Pensional es que se expide a en favor de:

1. Los afiliados que antes de la Ley 100 de 1993 hubieran realizado cotizaciones al ISS, Cajas de Previsión Social o Fondos de Previsión del sector público

2. Los afiliados que hubieran estado vinculados al Estado o sus entidades descentralizadas.

3. Las personas vinculadas por medio de un contrato laboral a empresas que tienen a su cargo el reconocimiento pensional y

4. Los afiliados que estuvieran afiliados a Cajas de Previsión Social del Sector Privado ${ }^{26}$.

Así mismo, si un afiliado al ISS estuvo vinculado con empleadores o empresas que asumían el reconocimiento de prestaciones sociales, al momento de trasladarse a alguno de los regímenes del SGP, se les emite un Título Pensional que tiene como finalidad que el servicio del trabajador sea reconocido en la HLP. En otras palabras, los títulos pensionales son pagarés emitidos por empresas privadas que tenían a su cargo el reconocimiento el pago de pensiones de sus "trabajadores, quienes, como afiliados obligatorios del régimen general de pensiones, escogieron el RPM" 27 se caracterizan por que la relación laboral tenía que estar vigente al momento de entrar en vigencia la Ley 100 de 1993. También son utilizados cuando el "empleador haya omitido la afiliación del trabajador al SGP" 28 .

Más adelante, a través del Decreto 1513 de $1998^{29}$, se establecieron los parámetros para la emisión de certificaciones con destino al cálculo de Bonos Pensionales A y B, porque cada entidad tenía su procedimiento interno y su propio formato de certificación. Posteriormente, el Ministerio de Hacienda y Crédito Público y el Ministerio de la Protección Social emiten la Circular Conjunta 13 de $2007^{30}$, que tenía finalidad como la de unificar los formatos de certificación para la emisión de Bonos pensionales, se elaboran tres formatos que componen la certificación laboral para la emisión de Bono Pensional, denominados Formatos CLEBP, que son la certificaciones de información laboral, salario base, y salarios mes a mes para la liquidación y emisión de Bonos Pensionales Tipo A.

Así mismo, el Ministerio de Protección Social — hoy Ministerio de Salud- emitió la Resolución 1303 de $2005^{31}$, por la cual estableció el mecanismo de recaudo denominado Planilla Integrada de Liquidación de Aportes, PILA, por el cual se buscó eliminar el diligenciamiento de formularios y facilitar el pago de los aportes mediante transferencias electrónicas, es decir, se tecnificó la manera de pagar los aportes a seguridad a través de una ventanilla virtual que permite la autoliquidación y pago de aportes al SGSS y parafiscales, es importante mencionar que en este sistema la responsabilidad por la veracidad e integridad de la información consignada es del aportante. 


\section{La historia laboral pensional unificada}

El Estado colombiano definió el diseño, organización y funcionamiento del Registro Único de los Afiliados (RUAF) ${ }^{32}$; es obligación de las AFP del SGSS alimentar el sistema. En este contexto, a partir de enero de 2008, Colpensiones implementó la unificación del sistema tradicional y la autoliquidación de aportes, en la cual se pueden ver los aportes efectuados desde 1967 a la fecha, lo cual se denomina Historia Laboral Unificada.

Posteriormente, Colpensiones creó la oficina virtual el Portal del Aportante, en la cual se les permite a las empresas conocer el estado de cuenta y corregir las inconsistencias en los reportes de pago. Al contrario, las AFP privadas que trabajan con cuentas de ahorro individual, la información es descentralizada no cuentan con un formato único, por lo cual si el afiliado se traslada de AFP, debe solicitar la información de su cuenta de ahorro en cada una de ellas.

Por otro lado, el Ministerio de Hacienda y Crédito Público y el Ministerio de la Protección Social emitieron la Circular Conjunta 13 de 2007, en la cual se adoptaron los formatos 1, 2 y 3 para la expedición de certificados físicos con destino a la emisión de bonos pensionales y/o para el reconocimiento de bonos pensionales. A continuación con la emisión de la Circular Conjunta 0065 de $2016^{33}$, el gobierno nacional buscó eliminar la confirmación de los tiempos públicos laborales anteriores a la entrada en vigencia de la Ley 100 de 1993; en consecuencia, las entidades que reconocen bonos o cuotas partes, garantizarán la veracidad de la información mediante una herramienta tecnológica que será administrada por la Oficina de Bonos Pensionales del Ministerio de Hacienda y Crédito Público, así se adoptará el Formato de Certificación Electrónica de Tiempos Laborados (CETIL).

\section{Construcción de la historia laboral pensional}

A partir de 1994, con la creación de SGP, el gobierno ha implementado varias estrategias para llevar un control sobre la afiliación y el pago de aportes de los ciudadanos al SGP, para poder cumplir con la obligación consagrada en la Constitución Nacional de "efectivizar la seguridad social en tanto (es una) garantía iusfundamental y servicio público de carácter obligatorio” ${ }^{34}$. En este capítulo se describe cómo se construye la HLP para efectos pensionales en Colombia. Para ello, se ilustra el proceso y se describen sus elementos y actores.

\section{Paso 1. Afiliación al Sistema General de Pensiones}

El primer paso de la construcción de la HLP es la afiliación al SGP, y esta es "la puerta de acceso al Sistema de Seguridad Social y, por lo tanto, se constituye en la fuente de los derechos y las obligaciones que ofrece o impone aquel. [...] Nadie puede predicar pertenencia al sistema de seguridad social, mientras no medie su afiliación; y ningún derecho o ninguna obligación de los previstos en dicho sistema se causa a su cargo sin la afiliación" 35 .

Consagrada en el artículo 15 de la Ley 100 de 1993, señala que la afiliación al SGP es obligatoria para “Todas aquellas personas vinculadas mediante contrato de trabajo o como servidores públicos. Así mismo, las personas naturales que presten directamente servicios al Estado o a las entidades o empresas del sector privado, bajo la modalidad de contratos de prestación de servicios, o cualquier otra modalidad de servicios que adopten, los trabajadores independientes y los grupos de población que por sus características o condiciones socioeconómicas sean elegidos para ser beneficiarios de subsidios a través del Fondo de Solidaridad Pensional, de acuerdo con las disponibilidades presupuestales" ${ }^{36}$. 
Debe entenderse aquí por "afiliación" el procedimiento para incorporar a cualquier individuo al SGP. Esta incorporación puede realizarse a cualquiera de los regímenes pensionales existentes: RPM o RAIS, y se "produce una vez en la vida del ciudadano, y que en esa medida no es un acto que sea susceptible de reiterarse en el tiempo" 37 . Las principales características de la afiliación es que es vitalicia, como está establecido en el artículo 13 del Decreto 692 de 1994, el cual señala: "La afiliación al SGP es permanente e independiente del régimen que seleccione el afiliado. Dicha afiliación no se pierde por haber dejado de cotizar uno o varios períodos, pero podrá pasar a la categoría de inactivos, cuando tenga más de seis meses de no pago de cotizaciones" 38 .

\section{Paso 2. Pago de aportes y reporte de novedades}

El segundo paso en la creación de la HLP es la cotización que "es una obligación eventual que nace bajo un determinado supuesto, como es la ejecución de una actividad en el mundo del trabajo o el despliegue de una actividad económica” 39 . La cotización al SGP es el pago del aporte y reporte de novedades. Para la vigencia 2017, la cotización al SGP corresponde al 16\% del ingreso base de cotización ${ }^{40}$. Esta cotización se examina en función del tipo de vinculación existente entre el trabajador y empleador, o para los contratistas e independientes, de conformidad con las siguientes características:

1. Trabajadores: para todas aquellas personas vinculadas mediante contrato de trabajo a una empresa, el aporte se distribuirá de la siguiente manera: el empleador paga el $12 \%$ y trabajador paga el $4 \%$ del ingreso base de cotización.

2. Servidores públicos: para los servidores públicos de entidades públicas con vinculación legal o reglamentaria con el Estado, el aporte se distribuirá de la siguiente manera el empleador paga el $12 \%$ y el trabajador paga el $4 \%$ del ingreso base de cotización.

3. Contratistas: las personas naturales que presten directamente servicios al Estado o a las entidades o empresas del sector privado, bajo la modalidad de contratos de prestación de servicios, o cualquier otra modalidad de servicios que adopten, es decir, mediante una vinculación civil o comercial con el Estado, cuyo aporte se calcula sobre la base del $40 \%$ del valor total del contrato facturado mensualmente, y cuya cotización es del 16\% para pensión está a cargo $100 \%$ del contratista y

4. Trabajadores independientes: a los trabajadores independientes por cuenta propia y los independientes con contrato diferente a prestación de servicios, tienen a cargo el $100 \%$ del porcentaje a cotizar para pensión, $16 \%$ sobre todos los ingresos.

El incumplimiento en el pago de la cotización, la mora en el pago de los mismos y la ausencia en el reporte de novedades son algunas de las causas que generan inconsistencias en la HLP del afiliado y afectan el reconocimiento de su derecho pensional. El pago de la cotización tiene sustento en el artículo 4 de la Ley 797 de 2003, que modifica el artículo 17 de la Ley 100 de 1993, y esta obligación termina una vez el afiliado reúne los requisitos para la pensión.

El incumplimiento en la obligación de girar el aporte al SGP origina la sanción descrita en el artículo 23 de la Ley 100 de 1993, que corresponde al interés moratorio que se cobra para el impuesto de renta y complementarios. En consecuencia de lo anterior, el artículo 24 de la Ley 100 de 1993 establece "Corresponde a las entidades administradoras de los diferentes regímenes adelantar las acciones de cobro con motivo del incumplimiento de las obligaciones del empleador de conformidad con la reglamentación que expida el gobierno nacional. Para tal efecto, la liquidación mediante la cual la administradora determine el valor adeudado, prestará mérito ejecutivo" ${ }^{41}$.

En este punto es importante señalar que las AFP deben aplicar los estándares establecidos por la Unidad de Gestión Pensional y Parafiscales ${ }^{42}$ (UGPP), porque las cotizaciones de aportes a pensión "son en 
realidad contribuciones parafiscales de destinación específica, en cuanto constituyen un gravamen, fruto de la soberanía fiscal del Estado, que se cobra obligatoriamente a determinadas personas para satisfacer sus necesidades de salud y pensiones y que, al no comportar una contraprestación equivalente al monto de la tarifa fijada, se destinan también a la financiación global (...) del Sistema General de Seguridad Social" ${ }^{43}$.

Así, las AFP del RPM utilizarán el cobro coactivo establecido en el artículo 57 de la Ley 100 de 1993 y reglamentado por el Decreto 2633 de $1994^{44}$, y las AFP del RAIS deben hacerlo por cobro ejecutivo 45 , siendo las AFP las obligadas a realizar el cobro a los empleadores morosos, siguiendo los parámetros establecidos por la UGPP. En consecuencia, si la AFP no cumple con los parámetros establecidos por la UGPP, pueden ser sancionados por esta entidad. Por otra parte, en la Sentencia T-398-13 ${ }^{46}$, la Corte Constitucional expone que la entidad de seguridad social que no ejerza las acciones de cobro, ni utilice los mecanismos judiciales establecidos en la Ley para que los empleadores cumplan con su obligación, se entenderá como allanada a la mora. Por tanto, la AFP será la obligada directa a reconocer el pago de la prestación, con fundamento en la omisión de su deber legal de cobro, lo cual busca proteger el derecho a la seguridad social de los afiliados.

\section{Paso 3. Liquidación de aportes y reporte de información}

El tercer paso en la creación de la HLP es la liquidación y posterior reporte de pago de aportes a pensión, o novedades en la Planilla Integrada de Liquidación de Aportes - PILA, manejado por los operadores de información ${ }^{47}$, que son entidades debidamente autorizadas, que generan la intermediación y/o conexión entre el aportante y PILA, administran el riesgo operativo y registran el pago de aportes y novedades previa inscripción por parte del empleador, de conformidad con el Decreto 1465 de $2005^{48}$.

Así como la Superintendencia Financiera de Colombia ejerce la inspección y vigilancia sobre la actividad realizada por los operadores de información que deben cumplir los requisitos establecidos en la Circular Externa 041 de $2007^{49}$, el empleador debe registrarse en cualquiera de estas entidades y a través de las mismas hacer el pago correspondiente. Si bien los operadores de información son canalizadores de la información y realizan la liquidación de aportes, su función se ha ampliado incluyendo procesos de validación; hoy constituyen un actor esencial del sistema.

Teniendo en cuenta que la liquidación de aporte al SGSS estaba sujeta a diversas interpretaciones por parte de los aportantes, operadores y las AFP, el Ministerio de Salud y Protección Social expidió la Resolución 2388 de 2016 con la finalidad de unificar y explicar las reglas para el recaudo de aportes. Así mismo, establece algunas operaciones de seguimiento con el propósito de generar alertas. Por ejemplo, respecto a los datos generales del aportante, establece: "El operador de información deberá notificar al aportante que no haya actualizado su información, por lo menos con dos (02) meses de anticipación al vencimiento de la fecha de actualización del registro y facilitará los mecanismos para la misma" 50 .

Además, establece procedimientos específicos de validación de la coherencia de los datos a cargo de los operadores y luego fija los pasos a seguir en caso de inconsistencias. En el caso de los aportes al SGP, el numeral 19 , título 7 , del capítulo 4 del anexo técnico 2, determina dos procedimientos de validación, para acceder a una pronta corrección de los errores y evitar con ello una recarga en la gestión de la validación. Este reporte permite una pronta gestión sobre las inconsistencias en la información de los trabajadores activos, pues evita posteriores incumplimientos sobre los aportes de los trabajadores y afectación en la HLP. 


\section{Paso 4. Consolidación y administración de la HLP}

El cuarto paso en la creación de la HLP es la consolidación de la información consignada por los aportantes y su posterior administración. En esta etapa, la AFP tiene conocimiento de las cotizaciones de cada uno de sus afiliados, para contabilizar el tiempo de servicio o el capital del afiliado y, en consecuencia, tiene el deber de custodia, conservación y guarda de la información de la HLP. Corresponde a las AFP, en el proceso de construcción de la HLP, consolidar para cada afiliado la información recibida a través de PILA con relación al pago de los aportes y el reporte de novedades. En consecuencia, cada AFP debe conocer las cotizaciones de sus afiliados, como insumo básico para contabilizar las semanas cotizadas y/o tiempos de servicio. La compilación de esta información da como resultado el insumo para la pensión: la HLP.

Para llegar a la adecuada y consistente consolidación de la HLP, la Ley 100 de 1993 otorga a las AFP determinadas facultades, como se ilustra en la Sentencia de la Corte Constitucional C-177-98, que señala: "Así, en particular, el artículo 53 de la Ley 100 de 1993 precisa que estas entidades [AFP] 'tienen amplias facultades de fiscalización e investigación sobre el empleador o agente retenedor de las cotizaciones al régimen, para asegurar el efectivo cumplimiento de la presente Ley', entre las cuales figura la posibilidad de: (i) Verificar la exactitud de las cotizaciones y aportes u otros informes; (ii) Adelantar las investigaciones que estimen convenientes para verificar la ocurrencia de hechos generadores de obligaciones no declarados; (iii) Citar o requerir a los empleadores o agentes retenedores de las cotizaciones al régimen, o a terceros, para que rindan informes; (iv) Exigir a los empleadores o agentes retenedores de las cotizaciones al régimen, o a terceros, la presentación de documentos o registros de operaciones, cuando unos u otros estén obligados a llevar libros registrados; (v) Ordenar la exhibición y examen de los libros, comprobantes y documentos del empleador o agente retenedor de las cotizaciones al régimen, y realizar las diligencias necesarias para la correcta y oportuna determinación de las obligaciones" ${ }^{51}$.

La Corte concluye que si la AFP no hace uso de las potestades que le otorga la ley y el empleador no le ha efectuado el traslado de los aportes del afiliado, la AFP no puede negar la pensión al afiliado ni trasladarle la responsabilidad de solicitar los aportes faltantes al empleador.

\section{Inconsistencias en la historia laboral pensional}

Con la expedición de la Resolución 2388 de 2016 del Ministerio de Salud y Protección Social, los operadores de información deben realizar operaciones de seguimiento para evitar el surgimiento de inconsistencias en la HLP de los afiliados y generar alertas oportunas. En este capítulo se muestran las inconsistencias que se pueden presentar en el proceso de construcción de la HLP y la forma de corregirlas en los dos regímenes: RPM y RAIS. Es importante identificarlas y corregirlas porque pueden obstaculizar el reconocimiento de la pensión.

Los principales Tipos de inconsistencias que se presentan en la HLP son:

\section{A. Problemas del proceso}

La primera inconsistencia que se tratara en este capítulo es el problema en el proceso, es decir, que en cada una de las etapas de construcción de la HLP, existe la posibilidad de generación de inconsistencias: En el paso 1 (Afiliación), los errores existentes provienen de la identificación del afiliado o del aportante, en el Paso 2 (Cotización) y en el Paso 3 (Liquidación de aportes y reporte de la información) la falta de pago de las cotizaciones por parte de los empleadores a las AFP, genera la denominada "mora por pago de aportes". 
1. Errores en identificación del aportante: en el paso 1 (Afiliación), los errores existentes provienen de la identificación del afiliado o del aportante. Por esta razón, en el proceso de afiliación al SGP, es importante determinar los tipos de aportantes: 1. Persona jurídica: cuando el aportante es una empresa a la cual presta sus servicios el afiliado y esta realiza los aportes a SGSS y 2. Persona natural: cuando el afiliado cotiza por sí mismo.

Las inconsistencias que se presentan en la etapa de afiliación, son: 1). Novedades por NIT. 2) Novedades por cédula o nombre del afiliado. 3) No existe afiliación al RPM, 4) Nombres no concuerdan con la Registraduría Nacional del Estado Civil. 5) Tipo de documento no concuerda con número de documento. 6) Afiliado trasladado al RAIS. 7) Multiafiliación. 8) Datos del afiliado no encontrado. 9) Registro no encontrado por afiliación, patronal, fecha y novedad y 10) Diligenciamiento errado en el formulario de autoliquidación.

2. Mora por pago de aportes: en los pasos 2 (Cotización) y 3 (Liquidación de aportes y reporte de la información), la falta de pago de las cotizaciones por parte de los empleadores a las AFP genera la denominada "mora por pago de aportes", caso en el cual podemos encontrarnos con alguna de estas tres (3) figuras:

- Deuda presunta: deuda aparente que se origina por omisión en el pago de aportes pensionales por un trabajador afiliado a un fondo de pensiones o por omisión en el reporte de novedades (i.e., retiro, cambios de salario, actualizaciones, traslados). Es una deuda que la AFP presume porque no se encuentra el pago por un ciudadano vinculado a la administradora y porque en el ciclo anterior no presenta novedad de retiro por parte del empleador, o porque el empleador realizó las consignaciones a otra AFP diferente a la que está afiliado el trabajador.

- Deuda real: deuda que se origina por pagos de aportes pensionales, realizados por un aportante, en los que se presentan errores o inconsistencias en la liquidación, o pagos realizados de manera extemporánea, en los que se omitió el pago de los correspondientes intereses de mora y,

- Convalidados: los convalidados no son deuda; son pagos de aportes pensionales, realizados por el aportante por trabajadores que no están afiliados a una AFP o a Colpensiones, o por los que no se registra relación laboral.

En el caso de presentarse mora en el pago de los aportes por parte del empleador, la AFP debe exigir al empleador el pago de los recursos, o el empleador o el trabajador pueden solicitar la corrección de la HLP.

\section{B. Consolidación y administración de las AFP}

En la consolidación y administración por parte de las AFP (paso 4) se presentan las siguientes inconsistencias: 1. Novedades no relacionadas. 2. Error en las correlaciones. 3. Falta de relación laboral. 4. Retiro retroactivo. 5. Afiliado fallecido. 6. Afiliado trasladado, entre otras.

1. Inconsistencias en la HLP el RPM: la HLP está compuesta por una parte administrativa y una parte financiera o de aportes. La corrección de esta es el proceso mediante el cual se analizan, depuran y actualizan los registros errados ya sea aportes y/o novedades. La mayoría de estos registros pueden ser tipificados, así: 1 . Registro no encontrado por afiliación, patronal, fecha y novedad. 2. Datos del afiliado no encontrado. 3. Inconsistencias en la migración de información de la base de datos autoliquidación a la base de datos SABASS. 4. Diligenciamiento errado en el formulario de autoliquidación. 5. Falta de información de novedades de pago por parte de los aportantes. 
Los errores en la HLP se clasifican en tres grupos:

- Período tradicional: son los errores presentados en el período de cotización comprendido entre el 1 enero de 1967 al 31 de diciembre de 1994. En este período se debe tener en cuenta el número patronal ${ }^{52}$ o el número de afiliación ${ }^{53}$ (tradicional o documento de identidad).

- Período actual: comprenden los errores presentados en el período de cotización del 1 de enero 1995 a la actualidad. En principio se usaron el formulario de liquidación de aportes y los cupones de pagos. Actualmente, el pago se realiza a través de PILA. En este período, la empresa o empleador se identifica con el NIT, cédula de ciudadanía, cédula de extranjería, entre otros y la afiliación es el número de identificación (cédula de ciudadanía, cédula de extranjería, etc.) del trabajador dependiente o independiente.

- Datos generales del solicitante: comprenden los errores que se presentan en el nombre, número de identificación, domicilio del afiliado.

En los informes presentados por la administradora del RPM, frente al cumplimiento del Auto 113 de 2014, señaló que el proceso de corrección contempla 3 grandes fases: Fase 1: Liquidación de la HLP y determinación de inconsistencias, Fase 2: Ejecución procesos masivos de corrección de inconsistencias y Fase 3: Realización manual de ajustes de inconsistencias no automatizadas ${ }^{54}$. Se identificaron errores desde el proceso de vinculación, como la múltiple afiliación que sólo es detectada al momento del reconocimiento de la prestación, del análisis en el RPM se identificaron las inconsistencias más recurrentes: 1. No existe afiliación al RPM. 2. No existe una relación laboral para el período pagado. 3. Nombres no concuerdan con Registraduría Nacional de la República. 4. Tipo de documento no concuerda con número de documento. 5 Afiliado fallecido. 6. Afiliado trasladado al RAIS. 7. Multiafiliación. 8. Régimen subsidiado. 9 Novedades no correlacionadas.

Para la corrección de las citadas inconsistencias, se han diseñado procesos como: 1. Homologación de nombres y número de documento. 2. Pagos con tipo de Documentos. 3. Pagos con tipo de documento en blanco o nulo. 4 Afiliación en cabeza del empleador. 5. Relaciones laborales, entre otras. El administrador del RPM afirma que ha identificado tres grandes problemas estructurales que inciden directamente sobre la falta de completitud de la HLP ${ }^{55}: 1$. Inconsistencias de la información que reposa en las bases de datos de Colpensiones. 2. Ausencia de un mecanismo unificado de información de los regímenes del SGP (RPM y RAIS). 3. Disponibilidad de la información de los tiempos cotizados en el sector público.

\subsection{Corrección de inconsistencias en el RPM:}

la corrección de inconsistencias en la HLP puede realizarse de tres formas: 1. Por petición del afiliado: mediante formulario radicado en los puntos de Atención de Colpensiones (PAC), Cades o Supercades, 2. Por parte del empleador en el portal web del aportante: el empleador puede corregir la HLP de sus empleados mediante el ingreso y ajuste en el portal citado y 3 . A través del portal web: el ciudadano puede consultar su HLP, y diligenciar el formulario electrónico de corrección de la HLP.

Una de las inconsistencias que más afectan a los afiliados y que generan no solo demoras en el estudio de la prestación, sino afectación en la consecución del reconocimiento, es la deuda de los empleadores a la AFP. La deuda ocasiona para los empleadores la carga de la obligación y la mora por el no pago a tiempo. Según informe de 2017 del administrador del RPM, la evolución de la deuda por concepto de cotizaciones a este régimen con corte al 31 de diciembre de 2016 , acumula un total de $\$ 663.848$ millones como deuda real y $\$ 16.236 .018$ millones como deuda presunta ${ }^{56}$. 


\section{Inconsistencias en la HLP del RAIS:}

en el RAIS, la pensión se construye con los aportes en las cuentas individuales de los afiliados y las correspondientes rentabilidades arrojadas durante los años en que efectuó sus aportes. Estos recursos (aportes y rentabilidades) constituyen el capital del cual se pagará la pensión del afiliado. Por lo anterior, al tratarse de cuentas individuales, las inconsistencias se centran en la deuda real y la deuda presunta. De la deuda que acumula el sistema pensional, uno de los grandes inconvenientes es que la mayor parte de la deuda es presunta y en consecuencia su cartera de difícil o imposible cobro y el valor que corresponde a deuda real se origina generalmente por mora, lo que deja un porcentaje a cartera con inexactitud y sin origen identificado.

Para corregir la falla estructural relacionada con la información de la HLP, en el caso de deuda en el pago de aportes al sistema pensional, la UGPP realiza el seguimiento y determinación de la liquidación y pago de las contribuciones parafiscales al sistema de la protección social ${ }^{57}$, en casos de inexactitud, mora u omisión en el pago de los aportes pensionales por parte de los empleadores, esta entidad establece los lineamientos que deben seguir las AFP en los procesos de cobro coactivo.

\section{Propuesta para mejorar el sistema de información de la historia laboral pensional del Distrito Capital 58}

\section{Centralización de la información de la historia laboral pensional distrital}

El proyecto de centralización de la HLP tiene como objetivo principal: "Poner a disposición de las entidades autorizadas y los ciudadanos, un aplicativo que controle los aportes a pensión de las entidades distritales, garantizando información correcta y oportuna, que permita generar una historia laboral sin inconsistencias". En esta aplicación, cada Entidad Distrital podrá verificar la HLP de sus trabajadores y sus novedades. En segundo lugar, se valida el oportuno y correcto pago de los aportes pensionales de los trabajadores. En tercer lugar, que los pagos que se hagan queden debidamente registrados por parte de las AFP. Respecto a la consolidación, esta se obtendrá a medida que se vayan verificando los aportes pensionales por cada uno de los trabajadores de forma mensual.

\section{Procedimiento para la Centralización de la información de la Historia Laboral Pensional Distrital}

1. Cargue de información por parte de las entidades: registro de los aportes pensionales y las novedades por parte del empleador, para lo cual se debe ingresar a la aplicación con usuario y contraseña, cargar el archivo (Se debe identificar el período), una vez cargada la aplicación procederá a la validación de la estructura de la misma.

2. Verificación de información con PILA: el FONCEP solicitará información al Ministerio de Protección Social con el fin de realizar la primera validación con PILA, en el cual se verificará el pago del aporte a pensión por parte de la entidad distrital empleadora. Una vez realizada esta validación, FONCEP definirá si requiere a la entidad empleadora por falta de pago o sigue con la segunda validación ante la AFP para comprobar que el pago quedó correctamente aplicado en la HLP en el respectivo período. En la información aportada por 1 PILA también debe hacerse una verificación del reporte de novedades. 
3. Verificación registro del aporte en la AFP: la segunda validación corresponde al registro del aporte o cotización en la AFP ya sea en el RPM o en el RAIS, esta validación permite llevar la trazabilidad del pago y asegurará que la cotización quede debidamente aplicada para el período contabilizando el número de semanas o aumentando la cuenta individual.

4. Consulta de HLP por parte del trabajador (activo o inactivo): el trabajador podrá ingresar a la aplicación a través de la página web de la entidad - FONCEP, previa verificación y validación, podrá solicitar la certificación de consistencia de la HLP de los tiempos laborados en el distrito. Si el trabajador está de acuerdo, puede imprimir esta certificación. Si el trabajador no está de acuerdo con la información, deberá solicitar verificación, a través del sistema de información indicando la inconsistencia que tiene la HLP.

5. Reporte de inconsistencias: la entidad que verifica y centraliza (i.e., FONCEP) deberá generar un reporte que arroje las inconsistencias existentes, con el fin de requerir a los empleadores distritales para que estos corrijan con la AFP estas inconsistencias, el papel de seguimiento se convierte en un mecanismo imprescindible para que no solo las inconsistencias se corrijan rápidamente, sino para generar al trabajador la confianza en la institucionalidad pública que debe velar por la eficiencia y eficacia administrativa en pro de los derechos de los ciudadanos.

6. Inconsistencias evidenciadas por el trabajador: pese a la creación de la herramienta de seguimiento mencionada, hay inconsistencias en la HLP que no son identificadas por este sistema, debido a que tanto la información del empleador como la registrada en las AFP es la misma, lo cual genera una aparente cohesión en la información; sin embargo, al ser revisada por el usuario o interesado final se evidencia un yerro desde el reporte de novedad o pago del empleador que es imposible identificar por la herramienta de seguimiento, verificación y consolidación.

\section{Generación de extractos}

La herramienta que se encuentra en implementación pretende generar extractos o estados de cada uno de los trabajadores del Distrito Capital para ser remitido a ellos de forma permanente y cumplir así la obligación de guarda y garantía de los derechos de los funcionarios como uno de los principios de la administración pública.
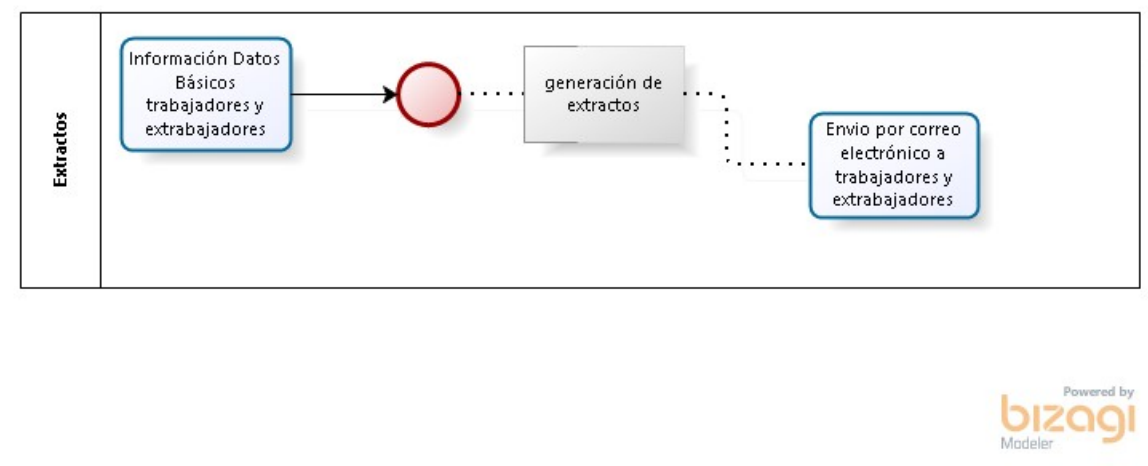

FIGURA 1

Certificación de la Historia Laboral Pensional

Fuente: estudio realizado en el marco del Convenio de Cooperación 000003 de 2016 celebrado entre el FONCEP y la OISS 

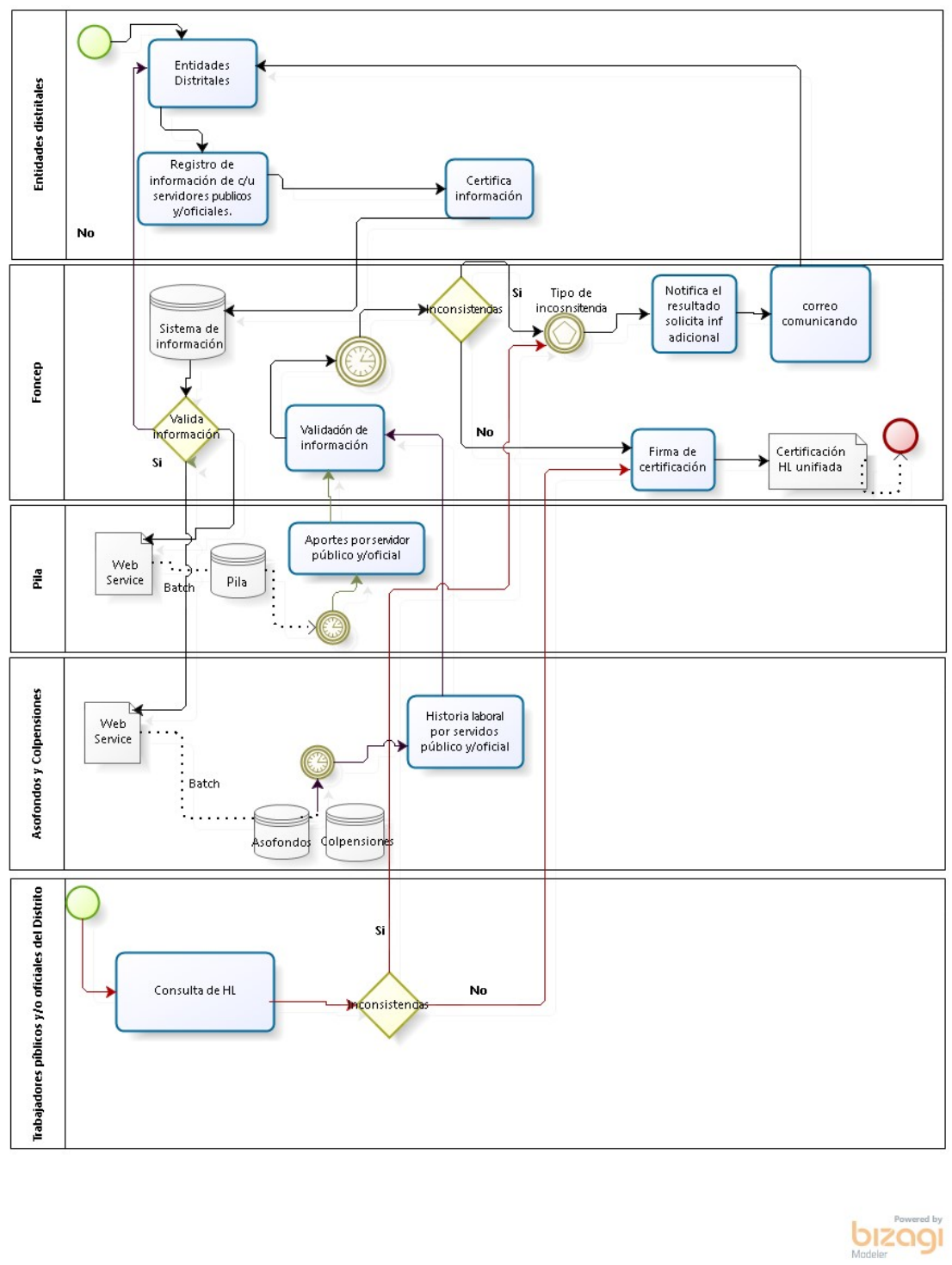

FIGURA 2

Flujo del proceso para la generación de la certificación de consistencia en la HLP

Fuente: estudio realizado en el marco del Convenio de Cooperación 000003 de 2016 celebrado entre el FONCEP y la OISS.

\section{Conclusiones}

- El sistema previsional anterior a la Ley 100 de 1993 era un sistema inequitativo, teniendo en cuenta que cada sector tenía su propia normatividad, y era improbable la acumulación de tiempos laborados para diferentes empleadores para el reconocimiento de la pensión, lo cual generó que pocas personas pudieran solicitar el reconocimiento de la pensión. Con la Ley 100 de 1993 se crearon el SGSS y el SGP, lo que permitió esta acumulación de tiempos laborados entre empleadores con la creación de los bonos pensionales y los títulos pensionales, con lo cual los afiliados pueden acceder a la pensión de vejez, invalidez o muerte.

- En el nuevo sistema impuesto por la Ley 100 de 1993, es responsabilidad de toda AFP la guarda, administración y conservación de la información de los afiliados. Quedó estipulado que, en los casos en que se presentan inconsistencia en la HLP, por falta de información o mora en los pagos, no debe afectarse al ciudadano cotizante y se invirtió la carga a las administradoras. La tecnificación de los 
sistemas de información relacionados con el SGP ha disminuido los errores en la HLP; no obstante, se siguen presentando inconsistencias que demoran el término de reconocimiento de las pensiones, por lo cual es necesario un sistema de centralización de la HLP.

- En el país es notoria la falta de cultura pensional entre los ciudadanos sobre la importancia de la HLP. Todo cotizante debe ser el primer auditor de la información consignada en su HLP o cuenta individual. Es importante que todos los ciudadanos revisen su HLP periódicamente, con el fin de detectar posibles inconsistencias y solucionarlas antes de solicitar el reconocimiento de la pensión. Para contribuir al proceso de sensibilización de los afiliados sobre la importancia de su HLP, se aconseja llevar a cabo un programa de fomento de la cultura pensional, que sea dirigido al ciudadano en general, para que los afiliados y sus familias se conviertan en los principales verificadores de la información allí consignada.

- Es axiomática la necesidad para el Estado de crear una herramienta que realice la verificación y consolidación de la HLP, al permitir a los ciudadanos consultar su HLP o cuenta individual, lo cual garantiza el deber de guarda y custodia de la información. Así mismo, esta herramienta deberá generar para las entidades la posibilidad de acciones correctivas cuando evidencien inconsistencias en los casos cuya responsabilidad se encuentre en cabeza de ellos, para prevenir su daño antijurídico. Esta herramienta deberá ser administrada por el Estado, en una entidad cuyo objeto se relacione con esta labor o que se cree de forma anexa a la gestión de cobro y seguimiento de la parafiscalidad.

\section{Bibliografía}

\section{Libros}

PONCE-BRAVO, GERMÁN ERNESTO, Seguridad social: tratado del régimen de prima media (Editorial Artemis, Bogotá, 2017).

\section{Revistas}

CASTILLO-CADENA, FERNANDO, Equidad pensional y salario base. La interpretación judicial actual como base de inequidad, 127 Vniversitas, 17-60 (2013). DOI: 10.1144/Javeriana.VJ127.epsb. Disponible en: http://www .scielo.org.co/pdf/vniv/n127/n127a02.pdf

\section{Informes}

Colpensiones, Informe Especial de Cumplimiento al Auto 113 de 2014 presentado a la Honorable Corte Constitucional, el 12 de junio de 2014 . Disponible en: http://www.corteconstitucional.gov.co/casocolpensiones/colpensiones/Informe\%20Especial\%20a\%20la\% 20H.\%20Corte\%20Constitucional\%20-\%20Auto\%20113\%20de\%202014.pdf

Colpensiones, Informe Especial presentado a la Corte en septiembre de 2015 para dar respuesta al Auto 181 de 2015. Disponible en: http://www.corteconstitucional.gov.co/casocolpensiones/colpensiones/Informe\%20Especial\% 20Auto\%20181\%20de\%202015.pdf

Colpensiones, Informe en respuesta al Auto 047 de 2017 de la Corte Constitucional. Disponible en: https://www.colpensiones.gov.co/Documentos/documentos_relacionados_al_seguimiento_de_la_corte_ constitucional/informes_presentados_a_la_hcc_por_colpensiones 


\section{Circulares y resoluciones}

Colombia, Ministerio de Hacienda y Crédito Público y el Ministerio de la Protección Social, Circular Conjunta 13 de 2007, 18 de abril de 2007 . Disponible en: http://www.minhacienda.gov.co/HomeMinhacienda/ShowProperty;jsessionid=02t2RoUniCgrt53U2X0 XInOqnl8KmvlRNTq6VWf5WRFsh1ZHPoFr!-89808455?nodeId=\%2FOCS\%2FMIG_5701219.PDF\%2 F\%2FidcPrimaryFile\&revision=latestreleased

Colombia, Ministerio de Hacienda y Crédito Público y el Ministerio de la Protección Social, Circular Conjunta 0065 de 2016, 17 de noviembre de 2016. Disponible en: http://www.minhacienda.gov.co/HomeMinhacienda/Show Property?nodeId=\%2FOCS\%2FP_MHCP_WCC-060648\%2F\%2FidcPrimaryFile\&revision=latestreleased

Colombia, Ministerio de Protección Social, Resolución 1303 de 2005, 11 de mayo de. Disponible en: https://www.m insalud.gov.co/sites/rid/Lists/BibliotecaDigital/RIDE/DE/DIJ/Resolucion-1303-de-2005.pdf

Colombia, Ministerio de Salud y de la Protección Social, Resolución 2388 de 2016, por la cual se unifican las reglas para el recaudo de aportes al Sistema de Seguridad Social Integral y Parafiscales, 10 de junio de 2016. Disponible en: https://www.minsalud.gov.co/sites/rid/Lists/BibliotecaDigital/RIDE/DE/DIJ/resolucion-2388-2016.pdf

Colombia, Superintendencia Financiera, Circular Externa 041 de 2007, 29 de junio de 2007. Disponible en: https:// m.superfinanciera.gov.co/descargas?com $=$ institucional\&name=pubFile21434\&downloadname=ce041_07.rtf

\section{Normatividad colombiana}

Colombia, Constitución Política, 116 Gaceta Constitucional, 20 de julio de 1991. Disponible en: http://www.secret ariasenado.gov.co/index.php/constitucion-politica

Colombia, Decreto 2633 de 1994, por el cual se reglamentan los artículos 24 y 57 de la Ley 100 de 1993, 41.620 Diario Oficial, 30 de noviembre de 1994. Disponible en: https://normativa.colpensiones.gov.co/colpens/docs/decreto _2633_1994.htm

Colombia, Decreto 1513 de 1998, por el cual se modifican y/o adicionan algunos artículos de los Decretos Reglamentarios 1748 de 1995 y 1474 de 1997 y se dictan otras disposiciones, 43.357 Diario Oficial, 6 de agosto de 1998. Disponible en: https://normativa.colpensiones.gov.co/colpens/docs/decreto_1513_1998.htm

Colombia, Decreto 1465 de 2005, por medio del cual se reglamentan los artículos 9 de la Ley 21 de 1982, el parágrafo 1 del artículo 1 de la Ley 89 de 1988, 287 de la Ley 100 de 1993, el numeral 4 del artículo 30 de la Ley 119 de 1994, 15 de la Ley 797 de 2003 y 10 de la Ley 828 de 2003, 45.905 Diario Oficial, 11 de mayo de 2005. Disponible en: http://www.alcaldiabogota.gov.co/sisjur/normas/Norma1.jsp?i=16499

Colombia, Decreto 3033 de 2013, por el cual se reglamentan los artículos 178 y 179 de la Ley 1607 de 2012 y se dictan otras disposiciones, 49.016 Diario Oficial, 27 de diciembre de 2013. Disponible en: http://www.alcaldiabogota .gov.co/sisjur/normas/Norma1.jsp?i=56254

Colombia, Ley 90 de 1946, por la cual se establece el seguro social obligatorio y se crea el Instituto Colombiano de Seguros Sociales, 26.322 Diario Oficial, 7 de enero de 1947. Disponible en: http://www.suin-juriscol.gov.co/v iewDocument.asp?id=1631247

Colombia, Ley 100 de 1993, por la cual se crea el sistema de seguridad social integral y se dictan otras disposiciones, 41.148 Diario Oficial, 23 de diciembre de 1993. Disponible en: http://www.secretariasenado.gov.co/senado/b asedoc/ley_0100_1993.html

Colombia, Ley 797 de 2003, por la cual se reforman algunas disposiciones del sistema general de pensiones previsto en la Ley 100 de 1993 y se adoptan disposiciones sobre los Regímenes Pensionales exceptuados y especiales, 45.079 Diario Oficial, 29 de enero de 2003. Disponible en: http://www.secretariasenado.gov.co/senado/basedoc/ley_ 0797_2003.html

Colombia, Ley 1607 de 2012, por la cual se expiden normas en materia tributaria y se dictan otras disposiciones, 48.655 Diario Oficial, 26 de diciembre de 2012. Disponible en: http://www.secretariasenado.gov.co/senado/basedoc/ ley_1607_2012.html 
Colombia, Ministerio de Gobierno, Decreto Ley 1600 de 1945, por el cual se organiza la Caja de Previsión Social de los Empleados y Obreros Nacionales, 25.893 Diario Oficial, 24 de julio de 1945. Disponible en: http://www.su in-juriscol.gov.co/viewDocument.asp?id=1864400

Colombia, Ministerio de Hacienda y Crédito Público, Decreto 692 de 1994, por el cual se reglamenta parcialmente la Ley 100 de 1993, 41.289 Diario Oficial, 30 de marzo de 1994. Disponible en: http://www.alcaldiabogota.go v.co/sisjur/normas/Norma1.jsp?i=6779

Colombia, Ministerio de Salud y Protección Social, Decreto 780 de 2016, por medio del cual se expide el Decreto Único Reglamentario del Sector Salud y Protección Social, 49.865 Diario Oficial, 6 de mayo de 2016. Disponible en: http://www.alcaldiabogota.gov.co/sisjur/normas/Norma1.jpp?i=65994

\section{Jurisprudencia}

Colombia, Corte Constitucional, Auto A-110-13, 5 de junio de 2013, magistrado sustanciador Luis Ernesto VargasSilva. Disponible en: http://www.corteconstitucional.gov.co/relatoria/autos/2013/a110-13.htm

Colombia, Corte Constitucional, Sentencia C-177-98, 4 de mayo de 1998, magistrado ponente Alejandro MartínezCaballero. Disponible en: http://www.corteconstitucional.gov.co/RELATORIA/1998/C-177-98.htm

Colombia, Corte Constitucional, Sentencia C-155-04, 24 de febrero de 2004, magistrado ponente Álvaro TafurGalvis. Disponible en: http://www.corteconstitucional.gov.co/relatoria/2004/C-155-04.htm

Colombia, Corte Constitucional, Sentencia SU-090-00, 2 de febrero de 2000, magistrado ponente Eduardo CifuentesMuñoz. Disponible en: http://www.corteconstitucional.gov.co/relatoria/2000/su090-00.htm

Colombia, Corte Constitucional, Sentencia T-068-98, 5 de marzo de 1998, magistrado ponente Alejandro MartínezCaballero. Disponible en: http://www.corteconstitucional.gov.co/relatoria/1998/T-068-98.htm

Colombia, Corte Constitucional, Sentencia T-398-13, 2 de julio de 2013, magistrado ponente Jorge Ignacio PreteltChaljub. Disponible en: http://www.corteconstitucional.gov.co/relatoria/2013/t-398-13.htm

Colombia, Corte Constitucional, Sentencia T-774-15, 18 de diciembre de 2015, magistrado ponente Luis Ernesto Vargas-Silva. Disponible en: http://www.corteconstitucional.gov.co/relatoria/2015/t-774-15.htm

Colombia, Corte Constitucional, Sentencia T-058-16, 12 de febrero de 2016, magistrado ponente Luis Guillermo Guerrero-Pérez. Disponible en: http://www.corteconstitucional.gov.co/relatoria/2016/t-058-16.htm

Colombia, Corte Constitucional, Sentencia T-079-16, 22 de febrero de 2016, magistrado ponente Luis Ernesto VargasSilva. Disponible en: http://www.corteconstitucional.gov.co/relatoria/2016/t-079-16.htm

Colombia, Corte Constitucional, Sentencia T-463-16, 29 de agosto de 2016, magistrada ponente Gloria Stella OrtizDelgado. Disponible en: http://www.corteconstitucional.gov.co/relatoria/2016/t-463-16.htm

Colombia, Corte Constitucional, Sentencia T-056-17, 3 de febrero de 2017, magistrado ponente Gabriel Eduardo Mendoza-Martelo. Disponible en: http://www.corteconstitucional.gov.co/relatoria/2017/t-056-17.htm

Corte Suprema de Justicia, Sala de Casación Laboral, Proceso 35211, 9 de septiembre de 2009, magistrado ponente Gustavo José Gnecco-Mendoza.

\section{Publicaciones electrónicas}

Disponible en: https://normativa.colpensiones.gov.co/colpens/docs/doc05.htm

\section{Notas}

1 Colombia, Constitución Política, 116 Gaceta Constitucional, 20 de julio de 1991, artículo 1.

2 Ibídem, art. 48. 
3 El Sistema de Seguridad Social Integral está conformado por los regímenes generales establecidos para pensiones, los servicios sociales complementarios, salud y riesgos profesionales.

4 Colombia, Ley 100 de 1993, por la cual se crea el sistema de seguridad social integral y se dictan otras disposiciones, 41.148 Diario Oficial, 23 de diciembre de 1993.

5 Colombia, Corte Constitucional, Sentencia T-463-16, 29 de agosto de 2016, magistrada ponente Gloria Stella OrtizDelgado.

6 Ibídem.

7 Colombia, Corte Constitucional, Sentencia T-079-16, 22 de febrero de 2016, magistrado ponente Luis Ernesto Vargas-Silva.

8 Colombia, Corte Constitucional, Sentencia T-058-16, 12 de febrero de 2016, magistrado ponente Luis Guillermo GuerreroPérez.

9 La Corte Constitucional dictamina que la información contenida en la HLP que se presente de manera errada, incompleta o inexacta, conlleva una grave vulneración por parte de las AFP del derecho al habeas data del trabajador. Colombia, Corte Constitucional, Sentencia T-463-16, 29 de agosto de 2016, magistrada ponente Gloria Stella Ortiz-Delgado.

10 Inconsistencia, en el caso de la HLP, hace referencia a que esta no está ajustada a la realidad o presenta errores.

11 Colombia, Corte Constitucional, Sentencia SU-090-00, 2 de febrero de 2000, magistrado ponente Eduardo CifuentesMuñoz. Define el ECI así: "se predica de aquellas situaciones en las que (1) se presenta una repetida violación de derechos fundamentales de muchas personas - que pueden entonces recurrir a la acción de tutela para obtener la defensa de sus derechos y colmar así los despachos judiciales - y (2) cuando la causa de esa vulneración no es imputable únicamente a la autoridad demandada, sino que reposa en factores estructurales. A hoy se ha declarado la existencia de ECI en el SGP en dos oportunidades. La primera vez mediante la Sentencia T-068-98, en la que se detectaron fallas de organización y procedimiento que afectaron la pronta resolución de solicitudes de reconocimiento y reliquidación de pensiones en la Caja Nacional de Previsión Social. La segunda, por medio del Auto A-110-13, evidenció que una de las fallas estructurales del SGP se desarrollaba en el sistema de información de la HLP del ISS en Liquidación y Colpensiones.

12 Semana de cotización: tiene un valor económico que es igual al aporte mensual convertido en términos semanales, lo que permite su acumulación en las cuentas de ahorro individual, y la generación de rendimientos, con miras a acceder a una pensión con base en el capital cuya cuantía depende del valor ahorrado, no del número de semanas cotizadas. FERNANDO CASTILLOCADENA, Equidad pensional y salario base. La interpretación judicial actual como base de inequidad, 127 Vniversitas, 17-60 (2013).

13 Colombia, Corte Constitucional, Sentencia C-177-98, 4 de mayo de 1998, magistrado ponente Alejandro MartínezCaballero.

14 Colombia, Ministerio de Gobierno, Decreto Ley 1600 de 1945, por el cual se organiza la Caja de Previsión Social de los Empleados y Obreros Nacionales, 25.893 Diario Oficial, 24 de julio de 1945.

15 Colombia, Ley 90 de 1946, por la cual se establece el seguro social obligatorio y se crea el Instituto Colombiano de Seguros Sociales, 26.322 Diario Oficial, 7 de enero de 1947. Años más tarde se llamó Instituto de Seguros Sociales (ISS).

16 En el sector público también se crearon diversos fondos o cajas de previsión social del nivel territorial, por entidades y hasta por sector económico (i.e. Caprecom, Caja de Previsión Social del Distrito, Foncolpuertos).

17 La historia laboral tradicional o sistema tradicional contenía la siguiente información: "1. Numero patronal: código de once dígitos que le asignaba el ISS a un patrono, el cual dependía de la ubicación geográfica y de la actividad económica que desarrollaba. (...) 2. Tipo de aportante: corresponde al código que identificaba el sistema de recaudo utilizado por el aportante para reportar sus novedades al ISS durante el período tradicional. (...) 3. Número de afiliación tradicional: código de nueve dígitos que identificaba a un afiliado ante el ISS. Existían dos clases de número de afiliación: - El que se asignaba dependiendo de la seccional y del número de orden (...) - El que se creaba con base en el número de documento e identidad del ciudadano que se afiliaba al ISS, los cuales podían ser: i. Afiliación cedula de ciudadanía; ii. Afiliación cedula de extranjería; iii. Afiliación tarjeta de identidad; iv. Afiliación NIT. 4. Tipo de afiliado: código numérico establecido por el ISS, que indicaba los seguros por los cuales un afiliado cotizaba al Sistema de Seguridad Social (pensión, salud y riesgos profesionales). En algunos casos dependía de la actividad económica de la empresa con la cual laboró. 5. Salario mensual base de cotización: se constituía sumando tres elementos (Decreto 3063 de 1989, artículo 79): - Parte física o básica; - Parte variable mensual no conocida; - Parte periódica no mensual conocida. 6. Exoneración: aplicaba 
para aquellos trabajadores que no estaban amparados por las contingencias propias de los seguros, por lo tanto no había lugar a la respectiva cotización. (...) 7. Información general: contenía: - Nombres y apellidos del afiliado; - Documentos de identidad del afiliado; - Fecha de nacimiento del afiliado; - Número de afiliaciones del afiliado; - Destino de reporte de semanas cotizadas: oficial o no válido para prestaciones económicas. 8. Relación de novedades registradas: se presentaba la información por cada uno de los empleadores, la cual a su vez discriminaba en: - Ingreso; - Cambio de salario; - Retiro". GERMÁN ERNESTO PONCE-BRAVO, Seguridad social: tratado del régimen de prima media, 74 (Editorial Artemis, Bogotá, 2017).

18 Colombia, Corte Constitucional, Sentencia C-177-98, 4 de mayo de 1998, magistrado ponente Alejandro MartínezCaballero.

19 Obligatoriedad de las cotizaciones: durante la vigencia de la relación laboral y del contrato de prestación de servicios, deberán efectuarse cotizaciones obligatorias a los regímenes del SGP por parte de los afiliados, los empleadores y contratistas con base en el salario o ingresos por prestación de servicios que aquellos devenguen”. Colombia, Ley 797 de 2003, por la cual se reforman algunas disposiciones del sistema general de pensiones previsto en la Ley 100 de 1993 y se adoptan disposiciones sobre los Regímenes Pensionales exceptuados y especiales, 45.079 Diario Oficial, 29 de enero de 2003, artículo 4.

20 Colombia, Ley 100 de 1993, por la cual se crea el sistema de seguridad social integral y se dictan otras disposiciones, 41.148 Diario Oficial, 23 de diciembre de 1993.

21 "Novedades comprende todo hecho que afecte el monto de las cotizaciones a cargo de los aportantes o de las obligaciones económicas que estos tienen frente al sistema. Las novedades pueden ser transitorias o permanentes: [las primeras] son las que afectan temporalmente el monto de las obligaciones económicas a cargo del aportante, tales como incapacidades, suspensiones del contrato de trabajo y variaciones no permanentes del Ingreso Base de Cotización... [y las segundas] son las que afectan la cotización base a cargo del aportante en relación con una determinada entidad administradora, tales como ingresos al sistema, cambios de empleador o retiro, traslado de entidad administradora y cambios permanentes en el Ingreso Base de Cotización, trabajadores dependientes al servicio de más de un patrono, cambio de condición de independiente a dependiente, o viceversa. Colombia, Ministerio de Salud y Protección Social, Decreto 780 de 2016, por medio del cual se expide el Decreto Único Reglamentario del Sector Salud y Protección Social, 49.865 Diario Oficial, 6 de mayo de 2016.

22 Colombia, Ministerio de Hacienda y Crédito Público, Decreto 692 de 1994, por el cual se reglamenta parcialmente la Ley 100 de 1993, 41.289 Diario Oficial, 30 de marzo de 1994.

23 En esta historia laboral se encontraba reflejada la información discriminada por cada empleador o por los aportes efectuados por el afiliado como trabajador independiente, mes a mes, año a año, la cual consistía en: Identificación del cotizante (empleador o independiente. Nombres y apellidos del cotizante (empleador o independiente). El ciclo pagado. La referencia del ciclo pagado (dependía del formulario de autoliquidación). La fecha de pago del ciclo. El documento de identidad del afiliado. Nombre y apellidos del afiliado. Novedades (retiros, traslados, licencias, etc.). Días cotizados. El ingreso base de cotización reportado. La tarifa pensional. El valor de cotización efectuado. En el sistema de Autoliquidación de aportes desaparecen todas las divisiones que contenía la HLP tradicional, y se ve "reflejada la información discriminada por cada empleador o por los aportes efectuados por el afiliado como trabajador independiente, mes a mes, año a año”. GERMÁN ERNESTO PONCE-BRAVO, Seguridad social: tratado del régimen de prima media, 85 (Editorial Artemis, Bogotá, 2017).

24 En la Sentencia T-056-17, la Corte Constitucional cita la definición de FERNANDO CASTILLO-CADENA, en la que señala que el Bono Pensional es "un valor a favor de un afiliado que se traslada a uno de los regímenes del SGP, el cual representa la deuda pensional causada desde el momento en que el afiliado inició su vida laboral hasta la fecha efectiva del traslado, en razón de las vinculaciones laborales, legales o reglamentarias que tuvo con las diferentes entidades de previsión que asumen el pago de la obligación". Y en este misma sentencia clasifica los Bonos Pensionales así: 1) De acuerdo con su emisor: el bono lo emite el último empleador o entidad pagadora de pensiones. 2) Dependiendo del régimen al cual se traslada el afiliado. 3) Los bonos especiales. Las clases de bonos pensionales son: Tipos de Bono pensionales: Tipo A: Se emiten a favor de personas que se trasladaron al RAIS; Tipo B: se emiten a favor de quienes se trasladan al RPM (empleados del Estado que se trasladaron al ISS al entrar en vigencia el SGP); Tipo C: A favor del Fondo de Previsión del Congreso y Tipo E: a favor de Ecopetrol. Tipo T: Exclusivos del RPM, que está destinado a cubrir la diferencia entre los dos regímenes pensionales aplicables a los servidores públicos antes de la entrada en vigencia del SGP y el régimen previsto para los afiliados al ISS con el fin de que el RPM pueda realizar el reconocimiento de una pensión con régimen de transición. https://normativa.colpensiones.gov.co/colpens/docs/doc05.htm

25 Colombia, Ley 100 de 1993, por la cual se crea el sistema de seguridad social integral y se dictan otras disposiciones, 41.148 Diario Oficial, 23 de diciembre de 1993. 
26 Colombia, Ley 100 de 1993, por la cual se crea el sistema de seguridad social integral y se dictan otras disposiciones, 41.148 Diario Oficial, 23 de diciembre de 1993.

27 GERMÁN ERNESTO PONCE-BRAVO, Seguridad social: tratado del régimen de prima media, 35 (Editorial Artemis, Bogotá, 2017).

28 Ibídem.

29 Colombia, Decreto 1513 de 1998, por el cual se modifican y/o adicionan algunos artículos de los Decretos Reglamentarios 1748 de 1995 y 1474 de 1997 y se dictan otras disposiciones, 43.357 Diario Oficial, 6 de agosto de 1998. Disponible en: https:// normativa.colpensiones.gov.co/colpens/docs/decreto_1513_1998.htm

30 Colombia, Ministerio de Hacienda y Crédito Público y el Ministerio de la Protección Social, Circular Conjunta 13 de 2007, 18 de abril de 2007.

31 Colombia, Ministerio de Protección Social, Resolución 1303 de 2005, 11 de mayo de 2005.

32 Que es un sistema de información que contiene el registro de los afiliados al SGSS. Colombia, Ley 797 de 2003, por la cual se reforman algunas disposiciones del sistema general de pensiones previsto en la Ley 100 de 1993 y se adoptan disposiciones sobre los Regímenes Pensionales exceptuados y especiales, 45.079 Diario Oficial, 29 de enero de 2003.

33 Colombia, Ministerio de Hacienda y Crédito Público y el Ministerio de la Protección Social, Circular Conjunta 0065 de 2016, 17 de noviembre de 2016.

34 Colombia, Corte Constitucional, Sentencia T-774-15, 18 de diciembre de 2015, magistrado ponente Luis Ernesto VargasSilva.

35 Corte Suprema de Justicia, Sala de Casación Laboral, Proceso 35211, 9 de septiembre de 2009, magistrado ponente Gustavo José Gnecco-Mendoza.

36 Colombia, Ley 797 de 2003, por la cual se reforman algunas disposiciones del sistema general de pensiones previsto en la Ley 100 de 1993 y se adoptan disposiciones sobre los Regímenes Pensionales exceptuados y especiales, 45.079 Diario Oficial, 29 de enero de 2003.

37 GERMÁN ERNESTO PONCE-BRAVO, Seguridad social: tratado del régimen de prima media, 14 (Editorial Artemis, Bogotá, 2017).

38 Colombia, Ministerio de Hacienda y Crédito Público, Decreto 692 de 1994, por el cual se reglamenta parcialmente la Ley 100 de 1993, 41.289 Diario Oficial, 30 de marzo de 1994.

39 Corte Suprema de Justicia. Sala de Casación Laboral, Proceso 35211. M.P. Gustavo José Gnecco Mendoza; 9 de septiembre de 2009.

40 Ejemplo: si el salario es de \$461.500 al mes, la cotización mínima que deben realizar un trabajador y su empleador a pensión obligatoria es de $\$ 73.840$ ( $16 \%$ del salario).

41 Colombia, Ley 100 de 1993, por la cual se crea el sistema de seguridad social integral y se dictan otras disposiciones, 41.148 Diario Oficial, 23 de diciembre de 1993.

42 La Unidad de Gestión Pensional y Parafiscales, UGPP, es una entidad del orden nacional, adscrita al Ministerio de Hacienda y Crédito Público, según la Ley 1607 de 2012, cumple la siguiente función: la UGPP será la entidad competente para adelantar las acciones de determinación y cobro de las Contribuciones Parafiscales de la Protección Social, respecto de los omisos e inexactos, sin que se requieran actuaciones persuasivas previas por parte de las administradoras. Parágrafo 1. Las administradoras del Sistema de la Protección Social continuarán adelantando las acciones de cobro de la mora registrada de sus afiliados, para tal efecto las administradoras estarán obligadas a aplicar los estándares de procesos que fije la UGPP. La UGPP conserva la facultad de adelantar el cobro sobre aquellos casos que considere conveniente adelantarlo directamente y de forma preferente, sin que esto implique que las administradoras se eximan de las responsabilidades fijadas legalmente por la omisión en el cobro de los aportes.

43 Colombia, Corte Constitucional, Sentencia C-155-04, 24 de febrero de 2004, magistrado ponente Álvaro Tafur-Galvis.

44 Colombia, Decreto 2633 de 1994, por el cual se reglamentan los artículos 24 y 57 de la Ley 100 de 1993, 41.620 Diario Oficial, 30 de noviembre de 1994. Disponible en: https://normativa.colpensiones.gov.co/colpens/docs/decreto_2633_1994.htm 
45 Colombia, Ley 100 de 1993, por la cual se crea el sistema de seguridad social integral y se dictan otras disposiciones, 41.148 Diario Oficial, 23 de diciembre de 1993.

46 Colombia, Corte Constitucional, Sentencia T-398-13, 2 de julio de 2013, magistrado ponente Jorge Ignacio PreteltChaljub.

47 Entre los operadores de información actualmente autorizados están: Asopagos, Banco Agrario, Compensar, Enlace Operativo, Fedecajas, Aportes en Línea, Pago Simple, Banco de Bogotá, Banco Popular, Banco Santander, Bancolombia, Citibank, Banco GNB Sudameris, BBVA, Banco de Crédito, Multibanca Colpatria, Banco de Occidente, BCSC, Davivienda y AV Villas.

48 Colombia, Decreto 1465 de 2005, por medio del cual se reglamentan los artículos 9 de la Ley 21 de 1982, el parágrafo 1 del artículo 1 de la Ley 89 de 1988, 287 de la Ley 100 de 1993, el numeral 4 del artículo 30 de la Ley 119 de 1994, 15 de la Ley 797 de 2003 y 10 de la Ley 828 de 2003, 45.905 Diario Oficial, 11 de mayo de 2005. Disponible en: http://www.alcaldiabogota.gov .co/sisjur/normas/Norma1.jsp?i=16499

49 Colombia, Superintendencia Financiera, Circular Externa 041 de 2007, 29 de junio de 2007.

50 Colombia, Ministerio de Salud y de la Protección Social, Resolución 2388 de 2016, por la cual se unifican las reglas para el recaudo de aportes al Sistema de Seguridad Social Integral y Parafiscales, 10 de junio de 2016.

51 Colombia, Corte Constitucional, Sentencia C-177-98, 4 de mayo de 1998, magistrado ponente Alejandro MartínezCaballero.

52 Código de 11 dígitos que asignaba el ISS a un patrono, dependía de la ubicación geográfica y de la actividad económica que desarrollaba.

53 Código de 9 dígitos que identificaba a un afiliado frente al ISS.

54 Colpensiones, Informe Especial de Cumplimiento al Auto 113 de 2014 presentado a la Honorable Corte Constitucional, el 12 de junio de 2014.

55 Colpensiones, Informe Especial presentado a la Corte en septiembre de 2015 para dar respuesta al Auto 181 de 2015.

56 Colpensiones, Informe en respuesta al Auto 047 de 2017 de la Corte Constitucional.

57 Contribuciones parafiscales del sistema de la protección social: se refieren a los aportes con destino al Sistema de Seguridad Social Integral conformado por el Sistema General de Seguridad Social en Salud, Pensiones y Riesgos Laborales, y a los establecidos con destino al Servicio Nacional de Aprendizaje (SENA), al Instituto Colombiano de Bienestar Familiar (ICBF) y al Régimen de Subsidio Familiar. Colombia, Decreto 3033 de 2013, por el cual se reglamentan los artículos 178 y 179 de la Ley 1607 de 2012 y se dictan otras disposiciones, 49.016 Diario Oficial, 27 de diciembre de 2013, artículo 1.

58 Esta sección está basada en el estudio Apoyo a la construcción del modelo administrativo y operativo para el manejo de los archivos y sistemas de información de la historia laboral del Distrito y la debida inspección, vigilancia y control frente a la oportuna liquidación y pago de las contribuciones parafiscales de la seguridad social. Definición de los procesos, plan de acción y socialización de la redefinición misional, realizado entre el Fondo de Prestaciones Económicas, Cesantías y Pensiones de Bogotá, FONCEP y la Organización Iberoamericana de Seguridad Social, OISS (2016).

\section{Licencia Creative Commons CC BY 4.0}

